\title{
Assessment of 3-T MRI using susceptibility-weighted imaging to detect and evaluate intra- or periarticular blood metabolites and meniscal tears of the knee
}

\author{
Dominik A. Sieron ${ }^{1 A, B, C, D, E, F, G}$, Dionysios Drakopoulos ${ }^{1 A, E, F}$, Milena Mitrakovic ${ }^{1 F}$, Marek Tombarkiewicz ${ }^{2 D}$, \\ Daniel Knap ${ }^{3 C, D}$, Ivan Platzek ${ }^{4 B, D, E}$, Maciej Tomalski ${ }^{5 A, B, F}$, Andreas Christe ${ }^{6 A}$
}

${ }^{1}$ Institute of Radiology and Neuroradiology, Tiefenau Hospital, Insel Group, Bern, Switzerland

${ }^{2}$ Department of Radiology, National Institute of Geriatrics, Rheumatology and Rehabilitation, Warsaw, Poland

${ }^{3}$ Departament of Radiology, Upper Silesia University Hospital, Katowice, Poland

${ }^{4}$ Department of Radiology, Dresden University Hospital, Dresden, Germany

${ }^{5}$ Medical University of Silesia, Katowice, Poland

${ }^{6}$ University Institute for Diagnostic, Interventional and Pediatric Radiology, University Hospital Bern, Switzerland

\section{Abstract}

Purpose: The purpose of this study was to assess the suitability of susceptibility-weighted imaging (SWI) sequences using the 3T MRI-unit for assessment of potential intraarticular pathologies in patients with acute and chronic torsion trauma of the knee joint.

Material and methods: Sixty-three patients with subacute and chronic rotary knee joint trauma of either the left or right knee were studied using an Achieva MRI 3T device (Philips, Amsterdam, Netherlands). Ground truth was set by two expert radiologists with seven and 10 years of experience in musculoskeletal imaging. Readings were performed separately for meniscus and joint space including synovia, ligaments, and periarticular soft tissue. Haemorrhage was defined as any lesion that was either T1 or SWI positive, without proton density (PD)-hypointensity (calcification). A lesion was defined as any pathology/variant with any signal positivity of either T1, PD, or SWI.

Results: A total of 63 patients were included ( $\mathrm{F}: \mathrm{M}=22: 41$ ). The median age of the cohort was 29 years (range 13 to 71 years). Thirty-nine patients showed a meniscal tear, and only three of them (7.7\%) demonstrated a meniscal haemorrhage. A total of 18 patients suffered from a periarticular injury, and 16 patients (88.9\%) demonstrated a concomitant periarticular haemorrhage.

Conclusions: These data suggest that SWI can be used for the diagnosis of intra- or periarticular blood metabolites because their potential have an impact on mechanical conflict with the surface of the knee joints, in particular the cartilage and their effect on malacic lesions, but it performs poorly in the detection of meniscal pathologies.

Key words: MRI imaging susceptibility, periarticular space, meniscus knee, haemorrhage.

\section{Introduction}

In recent years, magnetic resonance imaging (MRI) of the knee joint has been declared as the most sensitive and specific noninvasive method for evaluation of meniscal tears $[1,2]$. The routine protocol for evaluation of the knee entities includes conventional spin-echo T1-weighted image (T1WI), fast spin-echo proton density-weighted,

Correspondence address:

Maciej Tomalski, Medical University of Silesia, Katowice, Poland, e-mail: tomalmaciek@gmail.com

Authors' contribution:

A Study design · B Data collection · C Statistical analysis · D Data interpretation - E Manuscript preparation · F Literature search · G Funds collection 
and gradient-echo. The most used sequence for the meniscal inspection is a fast (or turbo) spin-echo, intermediate-weighted fat-suppressed sequence.

Susceptibility-weighted imaging (SWI) is now available on commercial MR scanners and is used widely in brain imaging. This sequence takes advantage of the difference in the magnetic susceptibilities of tissues, achieving a new type of contrast that differs from those obtained with conventional T1- and T2-weighted MR imaging [3]. SWI is extremely sensitive to changes of susceptibility and enhances the contrast between tissues with different susceptibilities. Studies that focus on its use in the knee-joint are lacking. SWI is by definition sensitive to paramagnetic susceptibility effects, in particular blood, calcification, haemosiderin, and slow flow in vascular structures [4]. Therefore, SWI is a potentially useful imaging tool in the demonstration of menisci, cartilage, subchondral bone, ligaments, and muscles. The aim of the present study was to compare SWI and pre-existing MRI techniques for detection of lesions, haemorrhage, and meniscal tear, particularly T1WI and proton density-weighted imaging.

\section{Material and methods}

\section{Data acquisition}

During the period from January to August 2017 we prospectively collected all posttraumatic cases of routine knee MRI in our institute. Inclusion criteria were the following: all patients who suffered a knee trauma of any kind, regardless of whether the cause was from a direct trauma or a rotatory accident. We included all age groups that were sent to us for assessment of post-traumatic consequences such as bone bruise, torn ligament, or meniscal tear. Exclusion criteria were the following: Patients with indication for knee MRI other than trauma, e.g. inflammation, infection, postoperative, rheumatoid arthritis, and bursitis. The MRI was performed on an Achieva MRI 3 Tesla unit (Philips, Amsterdam, Netherlands). For this continuously acquired representative population, we added an additional SWI transversal sequence, as a basic source, to the standard routine knee MRI protocol. VSWI, the $3 \mathrm{D}$ reconstructions based on the SWI transversal sequence, allowed calculation of the coronal and sagittal images. The entire MRI study consisted of a 26-minute protocol, with parameters shown in Table 1.

No contrast media was administered. Institutional Review Board (IRB) approval was waived due to the implementation of a well-established SWI clinical sequence into the clinical routine protocol.

\section{Ground truth}

Acquired MR images of knee joints were analysed by two radiologists with seven and 10 years of experience in musculoskeletal MRI imaging. For the ground truth, both readers had to agree on the signal intensity independently.

\section{Study}

Two additional general radiologists, with seven and three years of experience in musculoskeletal MRI imaging, reviewed the images in consensus for signal intensities.

The readout was performed on a GE-workstation (General Electric, Milwaukee, Wisconsin, USA) for two anatomical regions: 1) meniscus was analysed separately; and 2) the rest of the articular joint space including all ligaments (cruciate, collateral, patellar, and femoropatellar ligaments), synovia, capsule, and periarticular soft tissue.

All readers were blinded to the diagnosis and had to score the signal intensity on $\mathrm{T} 1$, proton density (PD), and SWI sequences for both anatomical regions separately. T1 and PD hyperintensity and SWI hypointensity in both regions were recorded and equalled positivity for haemorrhage. Any signal change (hyper- or hypointense) in any sequence was classified as positivity for any pathology (see Statistics below).

\section{Statistics}

A general lesion was defined as any pathology/variant with either PD, T1, or SWI hyper- or hypointensity.

Table 1. Magnetic resonance imaging examinations protocol

\begin{tabular}{|l|c|c|c|c|c|c|c|}
\hline Parameter & $\begin{array}{c}\text { PD SPAIR } \\
\text { coronal }\end{array}$ & $\begin{array}{c}\text { T1 TSE } \\
\text { coronal }\end{array}$ & $\begin{array}{c}\text { PD SPAIR } \\
\text { sagittal }\end{array}$ & $\begin{array}{c}\text { PD SPAIR } \\
\text { transversal }\end{array}$ & $\begin{array}{c}\text { T1 TSE } \\
\text { transversal }\end{array}$ & $\begin{array}{c}\text { PD VISTA SPAIR } \\
\text { sagittal }\end{array}$ & $\begin{array}{c}\text { SWI } \\
\text { transversal }\end{array}$ \\
\hline TR $(\mathrm{ms})$ & 3537 & 633 & Shortest & 6289 & 633 & 1300 & 31 \\
\hline TE $(\mathrm{ms})$ & 30 & 20 & 30 & 30 & 20 & 32 & TE1=7.2/delta TE $=6.2$ \\
\hline Slice thickness $(\mathrm{mm})$ & 3 & 3 & 3 & 3 & 3 & 0.7 & 2 \\
\hline Distance factor $(\%)$ & $0.3 \mathrm{~mm}$ & 0.3 & 0 & 0.3 & 0.3 & -0.35 & - \\
\hline FOV $(\mathrm{mm})$ & $160 \times 160$ & $160 \times 160$ & $160 \times 361$ & $160 \times 160$ & $170 \times 170$ & $140 \times 159$ & $230 \times 189$ \\
\hline Resolution & $320 \times 277$ & $384 \times 319$ & $160 \times 160$ & $292 \times 224$ & $380 \times 312$ & $200 \times 229$ & $384 \times 316$ \\
\hline Voxel size $(\mathrm{mm})$ & $0.5 \times 0.53$ & $0.417 \times 0.5$ & $0.55 \times 0.69$ & $0.55 \times 0.69$ & $0.45 \times 0.536$ & $0.7 \times 0.69$ & $0.6 \times 0.6$ \\
\hline
\end{tabular}

PD SPAIR - proton density-weighted imaging, T1 TSE - weighted imaging, SWI - susceptibility-weighted imaging 

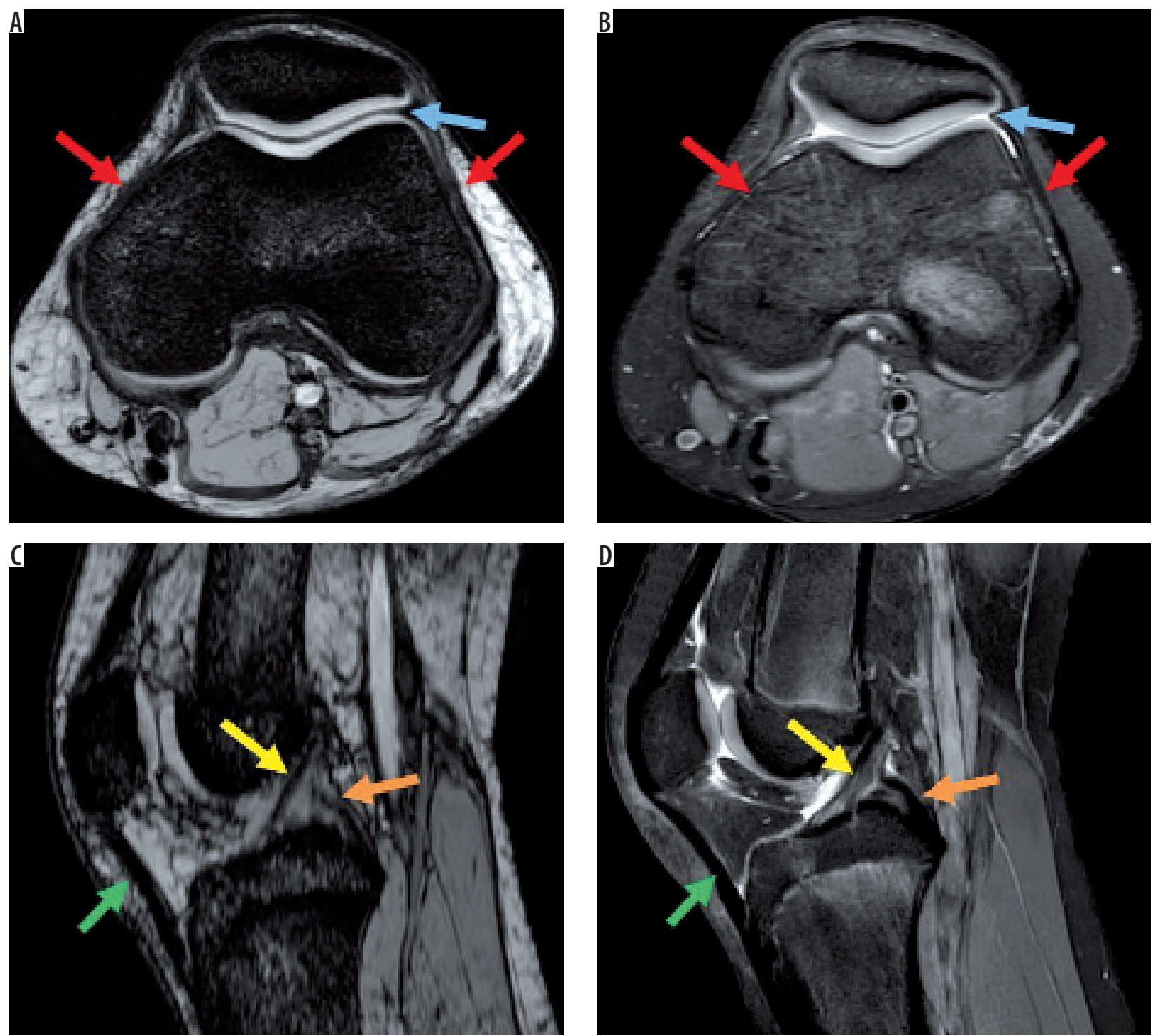

Figure 1. Magnetic resonance images showing normal anatomy. A) Axial susceptibility-weighted imaging (SWI) sequence without demonstrating haemorrhagic susceptibility artefacts. B) Corresponding proton density (PD)-weighted axial sequence with normal joint capsule (blue arrow) and retinaculum (red arrows). C) Sagittal SWI demonstrating normal anterior and posterior cruciate ligament (yellow and orange arrow) and patellar ligament (green arrow). D) Corresponding normal sagittal PD-weighted image

Haemorrhage in the periarticular space was defined as any lesion with either T1 hyperintensity or SWI hypointensity, without conspicuous PD-hypointensity (calcification). Meniscal haemorrhage was defined as T1 hyperintensity and SWI hypointensity, without conspicuous PD-hypointensity (calcification) (Figure 1).

Any signal change in any acquisition plane was defined as hyper- or hypointensity for the whole sequence. For the ground truth, both readers had to agree on positivity independently.

Sensitivity, specificity, and accuracy for haemorrhage and any lesion were calculated for T1, PD, and SWI, respectively, using the reader's detection rate against the ground truth. To compare significant differences of the sequences, Fisher's exact test was applied using MedCalc ${ }^{\circledast}$ version 7.6.0.0 (MedCalc Software, Mariakerke, Belgium). A significance level of $p<0.05$ was utilised.

\section{Results}

A total of 63 patients were included ( $\mathrm{F}: \mathrm{M}=22: 41)$. The median age of the cohort was 29 years (range 13 to 71 years). Thirty-nine patients showed a meniscal tear, and only three of them (7.7\%) demonstrated a meniscal haemorrhage (Figure 1). A total of 18 patients suffered from a periarticular injury, and 16 patients (88.9\%) demonstrated a concomitant periarticular haemorrhage (Figures 2 and 3 ).

\section{Periarticular joint space}

SWI demonstrates best sensitivity for detection of haemorrhage in the periarticular joint space: Sensitivity for T1, PD, and SWI was $25.0,62.5$, and $100 \%$, respectively (SWI vs. T1: $p<0.0001$ and SWI vs. PD: $p=0.018$ ). All three sequences scored specificities $>95 \%$, leading 

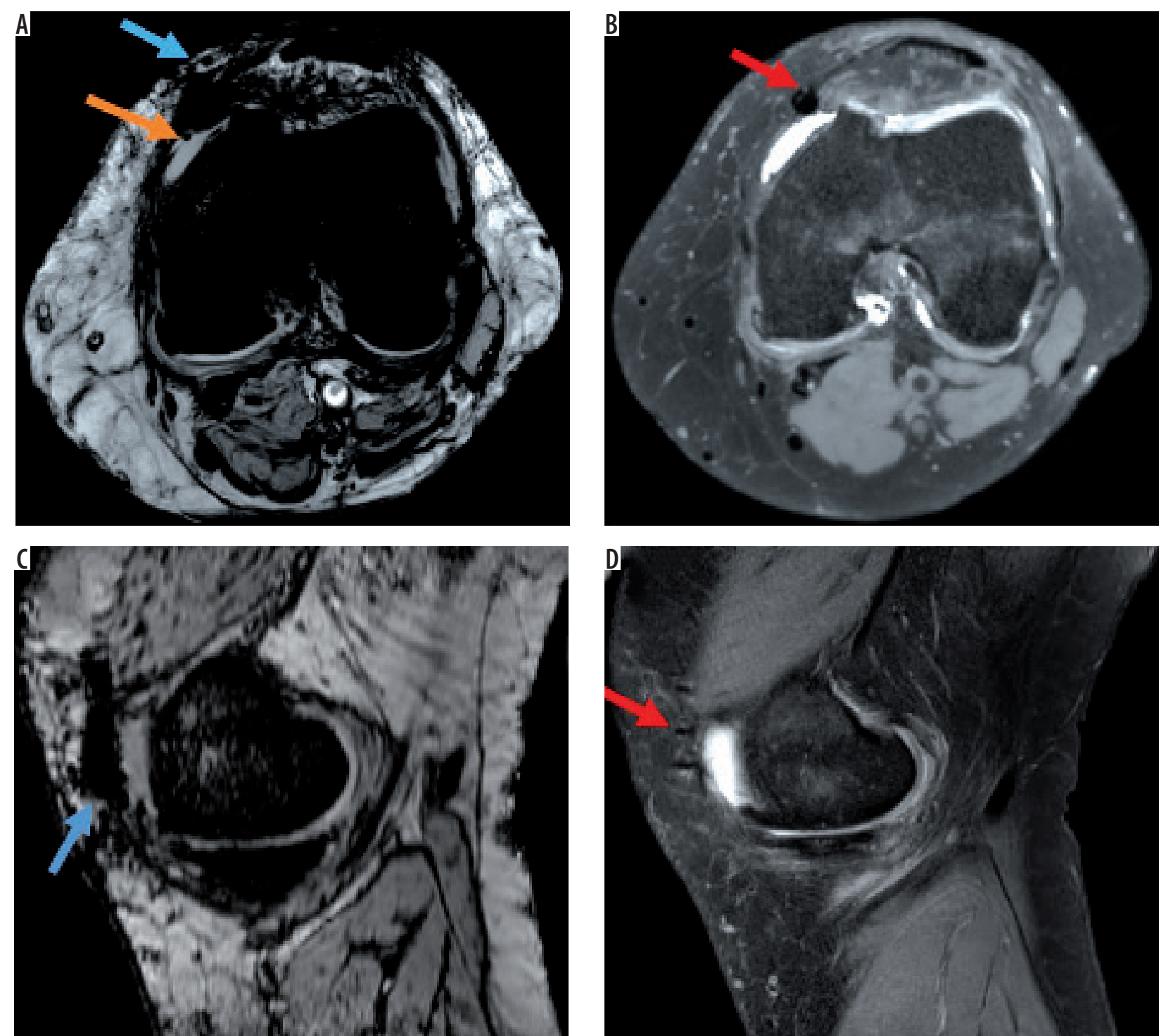

Figure 2. Post-traumatic case with intra- and periarticular haemorrhage. On A) axial susceptibility-weighted imaging (SWI) sequence and C) sagittal SWI excellent visibility of the periarticular haemorrhage with bold hypointensity in the anterior capsule (blue arrow) and intra-articular blood components (hypointense dots, orange arrow), B) corresponding axial proton density (PD)-weighted, and D) sagittal PD-weighted imaging without post-traumatic periarticular blood components. Postoperative changes (red arrows)

to the following accuracies for T1, PD, and SWI: 81.0 , 87.3 , and $96.8 \%$, respectively (SWI vs. T1: $p=0.009$ ). SWI also showed the best results for any lesion detection

Table 2. Sensitivity, specificity, and accuracy for periarticular pathology diagnostic in T1, proton density (PD), and susceptibility-weighted imaging (SWI) sequences

\begin{tabular}{|l|c|c|c|c|c|c|}
\multirow{2}{*}{ Pathology } & \multicolumn{6}{|c|}{ (Periarticular) Joint space } \\
\cline { 2 - 7 } & \multicolumn{3}{|c|}{ Haemorrhage } & \multicolumn{4}{c|}{ Any lesion } \\
\hline \multirow{2}{*}{ Sequence } & T1 & PD & SWI & T1 & PD & SWI \\
\cline { 2 - 7 } & $\%$ & $\%$ & $\%$ & $\%$ & $\%$ & $\%$ \\
\hline Sensitivity & 25.0 & 62.5 & 100.0 & 22.2 & 66.7 & 88.9 \\
\hline Specificity & 100.0 & 95.7 & 95.7 & 100.0 & 100.0 & 95.6 \\
\hline Accuracy & 81.0 & 87.3 & 96.8 & 77.8 & 90.5 & 93.7 \\
\hline
\end{tabular}

in the (peri)articular joint space: accuracy for T1, PD, and SWI was $77.8,90.2$, and $93.7 \%$, respectively (SWI vs. T1: $p=0.02$ and SWI vs. PD: $p=0.086$ ). Sensitivity of $\mathrm{T} 1$ to detect any lesion in the periarticular joint space was significantly lower for T1 $(22.2 \%)$ than that for PD $(66.7 \%, p=0.018)$ and SWI $(88.9 \%, p<0.0001)$ (Tables 2 and 3$)$.

\section{Meniscus}

Haemorrhage in the meniscus was best scored on SWI $(p<0.0001)$.

Accuracies for T1, PD, and SWI were 42.9, 34.9, and $98.4 \%$, respectively. While all sequences depicted meniscal haemorrhage (100\%), only SWI maintained a high specificity of $98.4 \%$; T1 and PD specificity dropped to $40 \%$ and $31.7 \%$, respectively. 

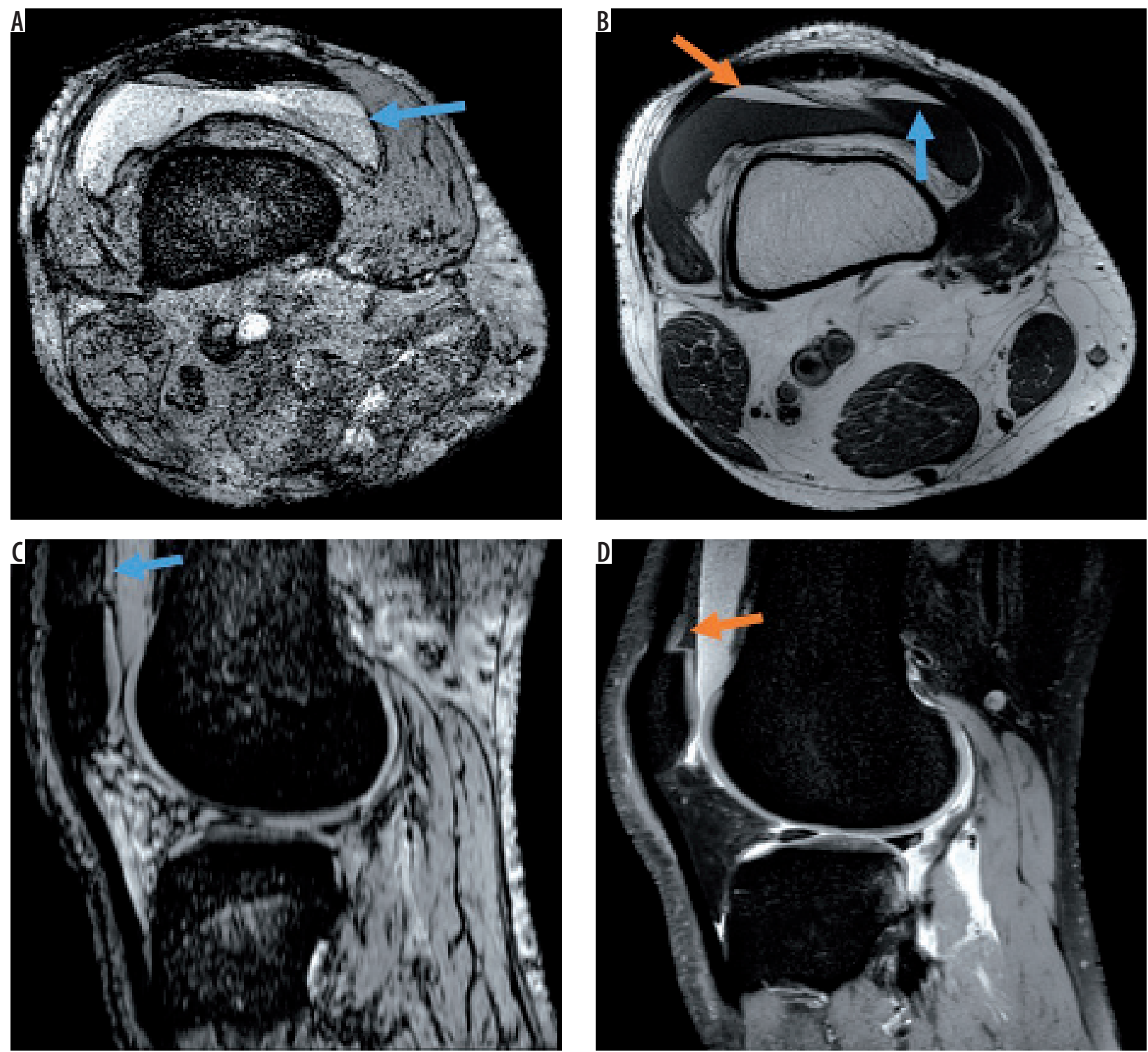

Figure 3. Post-traumatic haemorrhage displaying blood on all sequences. A) Axial susceptibility-weighted imaging (SWI) and C) sagittal SWI display intraarticular blood level with typical fat/fluid level (blue arrow) also visible on the B) axial T1-weighted image (hyperintense fat layer on top of fluid [orange arrow]) and corresponding hypointensity on the D) sagittal proton density-weighted image

Table 3. $P$-value board comparing sequences for joint space diagnostic in T1, proton density (PD), and susceptibility-weighted imaging (SWI) sequences

\begin{tabular}{|l|c|c|c|c|c|c|}
\hline \multirow{4}{*}{} & \multicolumn{3}{|c|}{ P-value board comparing sequences for joint space } \\
\cline { 2 - 7 } & \multicolumn{3}{|c|}{ Haemorrhage } & \multicolumn{3}{c|}{ Any lesion } \\
\cline { 2 - 7 } & VS. & T1 & PD. & vs. & T1 & PD \\
\hline \multirow{3}{*}{ Sensitivity } & SWI & $<0.0001$ & 0.018 & SWI & $<0.0001$ & 0.223 \\
\cline { 2 - 7 } & PD & 0.073 & PD & 0.018 & & \\
\hline Accuracy & SWI & 0.490 & 1.000 & SWI & 0.490 & 0.490 \\
\cline { 2 - 7 } & PD & 0.490 & PD & 1.000 & & \\
\cline { 2 - 7 } & SWI & 0.009 & 0.095 & SWI & 0.020 & 0.744 \\
\cline { 2 - 7 } & PD & 0.470 & PD & 0.086 & & \\
\hline
\end{tabular}

Furthermore, while SWI demonstrated a remarkable detection rate for haemorrhage and other lesions in the periarticular joint space, it demonstrated the lowest score for any meniscus lesions (Figure 3).
PD demonstrated the best accuracies for diagnosis of any lesion in the meniscus. The accuracy results for T1, PD, and SWI were $82.5,90.5$, and $44.4 \%$, respectively (Figure 2). The SWI accuracy was significantly lower than that of T1 and PD $(p<0.0001)$ (Tables 4 and 5).

\section{Discussion}

The purpose of our research was to apply SWI sequences in post-traumatic patients and to image blood components and their quantity within the structures of articular cavity and periarticular area of the knee joint. The study revealed great specificity and sensitivity of SWI sequences in assessing blood metabolites; however, it did not reveal an increased sensitivity in assessing post-traumatic changes within intra-articular structures, such as the ligament apparatus, especially the cruciate ligament and within the meniscus, in comparison with that of the standard protocol 

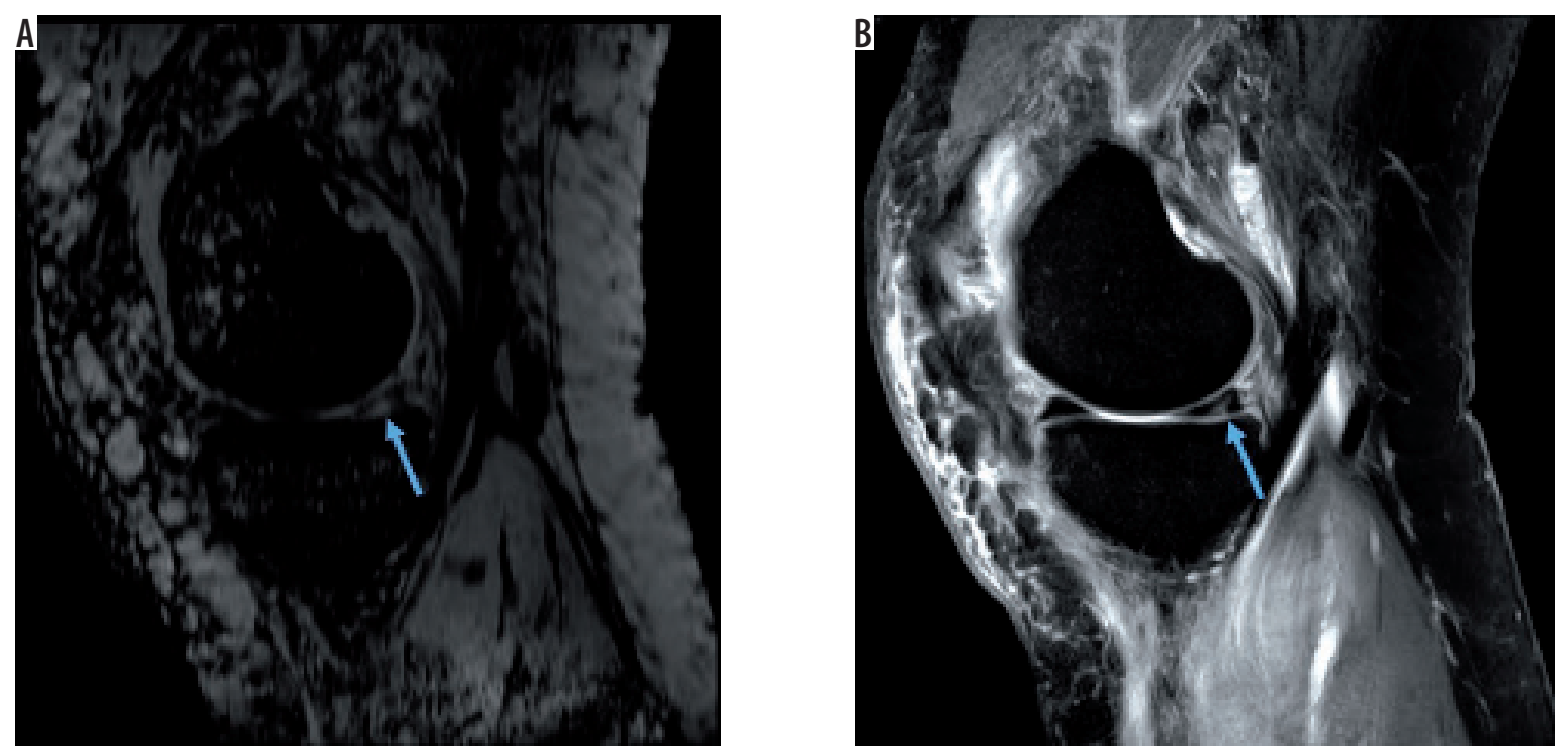

Figure 4. Hyperintense posterior meniscal tear without haemorrhage on A) sagittal susceptibility-weighted imaging and B) sagittal proton density-weighted image (blue arrows)

Table 4. Sensitivity, specificity, and accuracy for meniscal pathology diagnostic in T1, proton density (PD), and susceptibility-weighted imaging (SWI) sequences

\begin{tabular}{|l|c|c|c|c|c|c|}
\hline & \multicolumn{6}{|c}{ Meniscus } \\
\hline Pathology & \multicolumn{3}{|c}{ Haemorrhage } & \multicolumn{3}{c}{ Any lesion } \\
\hline \multirow{2}{*}{ Sequence } & T1 & PD & SWI & T1 & PD & SWI \\
\cline { 2 - 7 } & $\%$ & $\%$ & $\%$ & $\%$ & $\%$ & $\%$ \\
\hline Sensitivity & 100.0 & 100.0 & 100.0 & 87.2 & 100.0 & 10.3 \\
\hline Specificity & 40.0 & 31.7 & 98.3 & 75.0 & 75.0 & 100.0 \\
\hline Accuracy & 42.9 & 34.9 & 98.4 & 82.5 & 90.5 & 44.4 \\
\hline
\end{tabular}

used in the T1, T2, and PD imaging sequences [5-7]. As we know, MRI studies are performed in standard protocols without DWI because of the poor sensitivity and specificity for meniscal lesions and ACL trauma [8-11].

SWI protocol is a fairly new protocol that is successfully applied in neurology but not in orthopaedics. SWI is

Table 5. Sensitivity, specificity, and accuracy for meniscal pathology diagnostic in T1, proton density (PD), and susceptibility-weighted imaging (SWI) sequences

\begin{tabular}{|l|c|c|c|c|c|c|}
\hline \multirow{2}{*}{} & \multicolumn{4}{|c}{ P-value board comparing sequences for meniscus } \\
\cline { 2 - 7 } & \multicolumn{3}{|c}{ Haemorrhage } & \multicolumn{3}{c|}{ Any lesion } \\
\cline { 2 - 7 } & vs. & T1 & PD & vs. & T1 & PD \\
\hline \multirow{3}{*}{ Sensitivity } & SWI & 1.000 & 1.000 & SWI & $<0.0001$ & $<0.0001$ \\
\cline { 2 - 7 } & PD & 1.000 & & PD & 0.055 & \\
\hline Accuracy & SWI & $<0.0001$ & $<0.0001$ & SWI & 0.050 & 0.050 \\
\cline { 2 - 7 } & SD & 0.447 & & PD & 1.000 & \\
\cline { 2 - 7 } & PD & 0.465 & & PD & 0.271 & \\
\hline
\end{tabular}

used during routine brain diagnostics, e.g. in diagnosing and assessing intracranial brain haemorrhage or calcification or in assessing the image of cerebral veins in the case of an increase in the deoxyhaemoglobin level $[1,2,12]$.

Magnetic resonance has become the routine examination in assessing knee joint structures as a diagnostic examination and often as an examination that precedes surgery planning [3,13-15]. In fact, this is the reason why the assessment of quantity of blood metabolites within the knee joint, in the case of patients with chronic injury, may have clinical significance in terms of the influence of intra-articular microcalcification on the articular surface of the knee joint and possible microtrauma. During MRI examination performed soon after an injury, intra-articular fluid and fresh blood are often visible. During medical check-up, often performed after 4-6 weeks due to the waiting time for a medical procedure, blood and its metabolites are no longer visible with standard sequences.

Blood metabolites were also invisible in basic sequences within soft tissues in the knee joint area. Such metabolites, observed in imaging, may directly affect patients' complaints.

Among available articles, only one study describes the application of the SWI protocol for assessing structures within the knee, such as meniscal structures [16]. In our view, the SWI protocol is not very sensitive and specific and makes it possible to overlook smaller changes in comparison with that for standard T1, T2, and PD sequences. The SWI protocol does not allow accurate assessment of the course of meniscal structure injury, which is essential in planning for arthroscopic surgery [16].

The SWI protocol does not exhibit appropriate sensitivity or specificity in diagnosing ACL injury. In our study, we did not obtain positive images of ACL injury when compared with those from standard MRI sequences [17]. 
We also did not visualise blood metabolites within injured ACL tendons.

Arthroscopic surgery of the knee joint is a gold standard in assessing intra-articular structures; however, routine examinations to date, such as MRI, constitute a diagnostic routine and may often have an influence on the decision concerning patient's surgery, which is reflected in the patient's quality of life and has an economic and diagnostic impact $[7,18]$. An experienced physician is able to assess meniscal or ligament apparatus injury based on clinical examination just as well as via diagnostic imaging. However, physicians are unable to determine the quality and quantity of blood metabolites within the knee joint before and after surgery, which may have an influence on microtrauma of articular cartilage of the knee joint.

We are aware that when examining patients' knee joints using MRI, the SWI sequence is not fundamental. However, the SWI sequence allows us to obtain additional information, which we have previously described, within the cartilage.
The sequence also allows the detection of possible venous malformations, intra-articular haemorrhage, early degenerative changes of calcific nature, or venous malformations.

\section{Conclusions}

SWI can be used to diagnose intra- and periarticular blood metabolic detection, but its sequence fails to detect meniscal tears according to the present study.

\section{Ethical standards and informed consent}

Ethical approval (IRB) and informed consent were waived due to the retrospective nature of the study with irreversible anonymisation of patient identifiers.

\section{Conflict of interest}

The authors declare no conflict of interest.

\section{References}

1. Crawford R, Walley G, Bridgman S, Maffulli N. Magnetic resonance imaging versus arthroscopy in the diagnosis of knee pathology, concentrating on meniscal lesions and ACL tears: a systematic review. Br Med Bull 2007; 84: 5-23.

2. Crues JV, Mink J, Levy TL, Lotysch M, Stoller DW. Meniscal tears of the knee: accuracy of MR imaging. Radiology 1987; 164: 445-448.

3. Fowler PJ, Regan WD. The patient with symptomatic chronic anterior cruciate ligament insufficiency. Results of minimal arthroscopic surgery and rehabilitation. Am J Sports Med 1987; 15: 321-325.

4. Gasparotti R, Pinelli L, Liserre R. New MR sequences in daily practice: susceptibility weighted imaging. A pictorial essay. Insights Imaging 2011; 2: 335-347.

5. Khoury NJ, Mahfoud Z, Masrouha KZ, et al. Value of sagittal fat-suppressed proton-density fast-spin-echo of the knee joint as a limited protocol in evaluating internal knee derangements. J Comput Assist Tomogr 2011; 35: 653-661.

6. Matiotti SB, Soder RB, Becker RG, Santos FS, Baldisserotto M. MRI of the knees in asymptomatic adolescent soccer players: a casecontrol study. J Magn Reson Imaging 2017; 45: 59-65.

7. Yan R, Wang H, Yang Z, Ji ZH, Guo YM. Predicted probability of meniscus tears: comparing history and physical examination with MRI. Swiss Med Wkly 2011; 141: w13314.

8. Alaia EF, Benedick A, Obuchowski NA, et al. Comparison of a fast 5-min knee MRI protocol with a standard knee MRI protocol: a multi-institutional multi-reader study. Skeletal Radiol 2018; 47: $107-116$

9. Blake MH, Lattermann C, Johnson DL. MRI and arthroscopic evaluation of meniscal injuries. Sports Med Arthrosc Rev 2017; 25: 219-226.

10. Sparacia G, Barbiera F, Bartolotta TV, Midiri M, De Maria M, Lagalla R. Pitfalls and limitations of magnetic resonance imaging in bucket-handle tears of knee menisci. Radiol Med 2002; 104: 150-156.
11. Van Dyck P, Vanhoenacker FM, Lambrecht V, et al. Prospective comparison of 1.5 and 3.0-T MRI for evaluating the knee menisci and ACL. J Bone Joint Surg Am 2013; 95: 916-924.

12. Mittal S, Wu Z, Neelavalli J, Haacke EM. Susceptibility-weighted imaging: technical aspects and clinical applications, part 2. AJNR Am J Neuroradiol 2009; 30: 232-252.

13. Esmaili JAA, Keyhani S, Zarei R, Moghaddam AK. Accuracy of MRI in comparison with clinical and arthroscopic findings in ligamentous and meniscal injuries of the knee. Acta Orthop Belg 2005; 71: 189-196.

14. Fabricant PD, Yen YM, Kramer DE, et al. Fixation of Traumatic chondral-only fragments of the knee in pediatric and adolescent athletes: a retrospective multicenter report. Orthop J Sports Med 2018; 6: 2325967117753140.

15. Liu C, Liu C, Si L, Shen H, Wang Q, Yao W. Relationship between subchondral bone microstructure and articular cartilage in the osteoarthritic knee using 3T MRI. J Magn Reson Imaging 2018; doi: 10.1002/jmri.25982 [Epub ahead of print].

16. Chen W, Zhao J, Wen Y, et al. Accuracy of 3-T MRI using susceptibility-weighted imaging to detect meniscal tears of the knee. Knee Surg Sports Traumatol Arthrosc 2015; 23: 198-204.

17. Camarda L, Grassedonio E, Albano D, Galia M, Midiri M, D’Arienzo M. MRI evaluation to predict tendon size for knee ligament reconstruction. Muscles Ligaments Tendons J 2017; 7: 478-484.

18. Weinstabl R, Muellner T, Vecsei V, Kainberger F, Kramer M. Economic considerations for the diagnosis and therapy of meniscal lesions: can magnetic resonance imaging help reduce the expense? World J Surg 1997; 21: 363-368. 